\title{
Brief Description for the Development of a Protocol for the Aseptic Culture of Quercus Robur
}

ISSN: 2637-7659

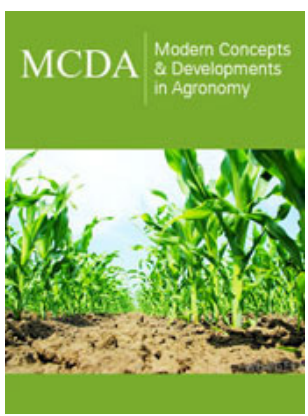

*Corresponding author: Paola Chiavazza, Department of Agricultural, Forestry and Food Sciences (DISAFA), ULF Agronomy, Università degli Studi di Torino, Largo Paolo Braccini, 2 (formerly Via Leonardo da Vinci, 44), 10095 GRUGLIASCO (TO), Italy

Submission: 望 February 10, 2020

Published: 侮 February 12, 2020

Volume 6 - Issue 1

How to cite this article:

Sara Sozzo, Antonio Nosenzo, Paola Chiavazza. Brief Description for the Development of a Protocol for the Aseptic Culture of Quercus Robur. Mod Concep Dev Agrono.6(1). MCDA.000626.2020.

DOI: 10.31031/MCDA.2020.06.000626

Copyright@ Paola Chiavazza, This article is distributed under the terms of the Creative Commons Attribution 4.0 International License, which permits unrestricted use and redistribution provided that the original author and source are credited.

\section{Sara Sozzo, Antonio Nosenzo and Paola Chiavazza*}

Department of Agricultural, Forestry and Food Sciences (DISAFA), Università degli Studi di Torino, Italy

\section{Opinion}

Experiments are underway, at the DISAFA in vitro culture laboratory, aimed at testing the optimal conditions for the recovery of Quercus robur samples suitable for restocking and developing in vitro multiplication protocols for selected abiotic stress-resistant genotypes (Figure 1). The germplasm taken into consideration consists of zygotic buds and embryos for which it is essential to be able, initially, to define the ideal conditions of vitality during growth and to optimize the culture conditions for the resumption of development. Nodal and apical segments of Quercus robur bearing buds have been taken both from young plants, obtained from seed and grown in pots on soil and from adult plants. The buds were placed in culture on a special WPM substrate, added with appropriate plant growth regulators (IAA $0.5 \mathrm{mg} / \mathrm{l}$, kinetin $1 \mathrm{mg} / \mathrm{l}$ ) and kept in a growth chamber at constant temperature of $25^{\circ} \mathrm{C}$, with a photoperiod of $16 \mathrm{~h}$. The first times of culture were characterized by extensive pollution due to endogenous bacteria and fungi of various kinds (Figure 2). With a close succession of subcultures, the sudden elimination of polluted subjects and the use of Plant Preservative Medium, a mixture of bactericidal/fungicidal agents added to the culture medium, it has been possible to contain pollution around $10 \%$ (Figure 3).

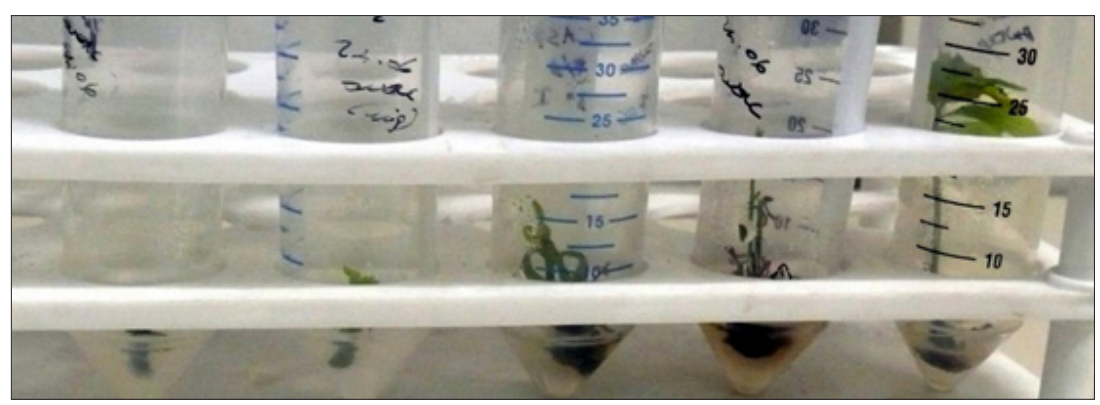

Figure 1: Explants of Quercus robur at different times of the multiplication cycle.

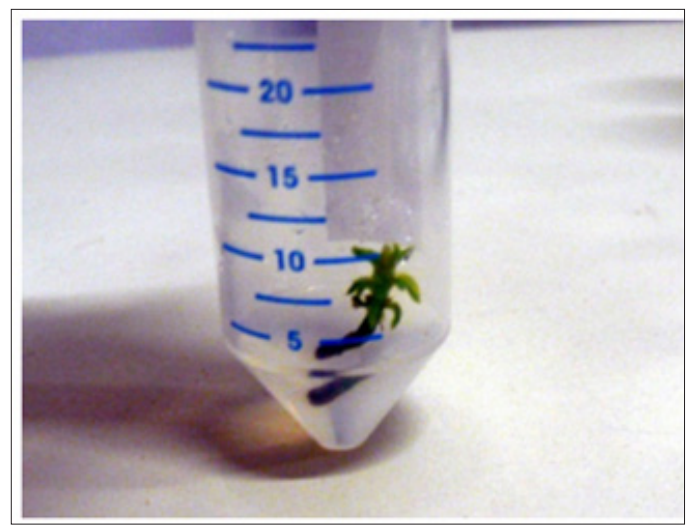

Figure 2: Q. robur explant at 15 days. 


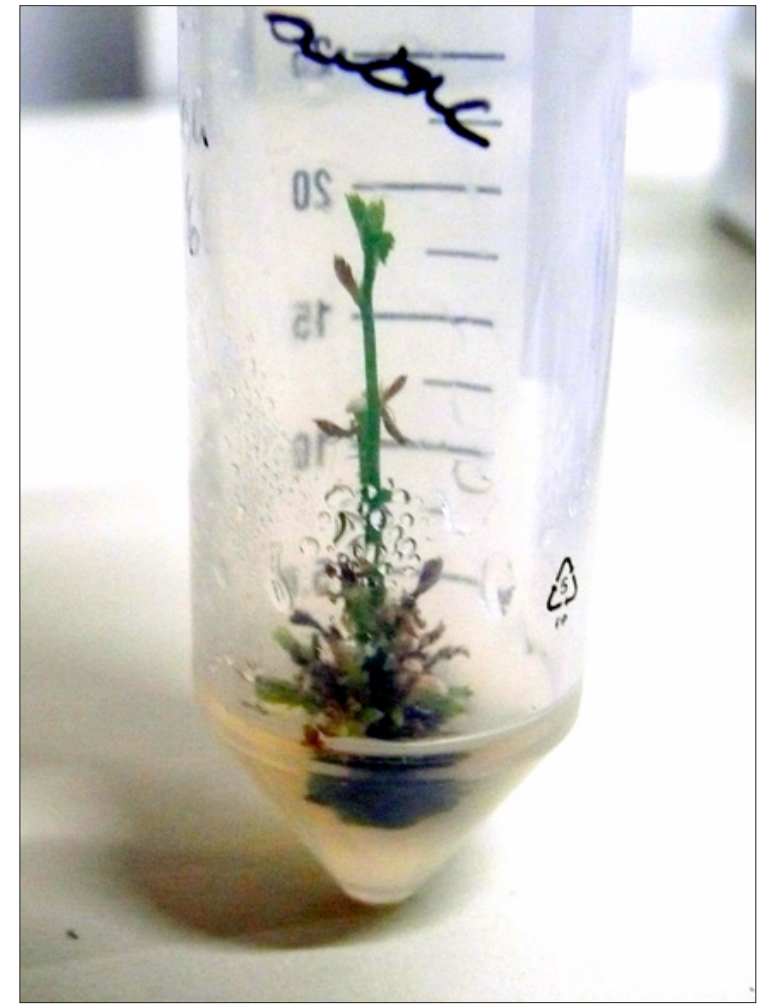

Figure 3: Elongation of several bud apices, one of which is the main.

About $20 \%$ of the buds taken from an adult plant developed producing stalks, compared to $40 \%$ of the buds taken from plants grown in soil. At present, about $20 \%$ of the buds taken from plants grown in pots have given rise to elongation with the relative production of new knots. When multiple stems develop from the same bud, usually only one continues to stretch, while the rest degenerate. Growth is still slow (about $1 \mathrm{~cm}$ in height in 20-30 days) (Figure 4).The buds were also used to test the influence that endogenous hormone levels can exert in the sprout development phase. For this purpose, the buds were placed in culture, separately, according to their position. Significant differences have been observed between the basal buds, which form callus and do not give rise to the formation of stems and the apical and sub-apical buds, which produce little callus and develop less slowly (Figure 5). There are ongoing tests to increase auxin concentration (IAA $0.75 \mathrm{mg} / \mathrm{l}$ ), to try to give rise to a faster stretch. An alternative propagation technique would also be able to obtain somatic embryogenesis from adult tissue. For this purpose, young leaves were cultured on a medium with the addition of cytokinin (kinetin $2 \mathrm{~m} / \mathrm{l}$ ). The achievement of somatic embryos would allow to remedy to an other moment of the in vitro culture considered critical, the formation of roots.

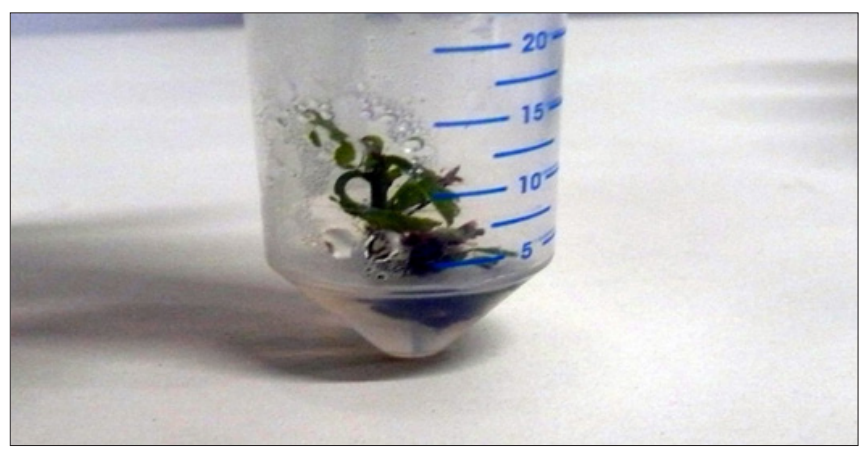

Figure 4: Head of $Q$. robur explants at 25 days of age.

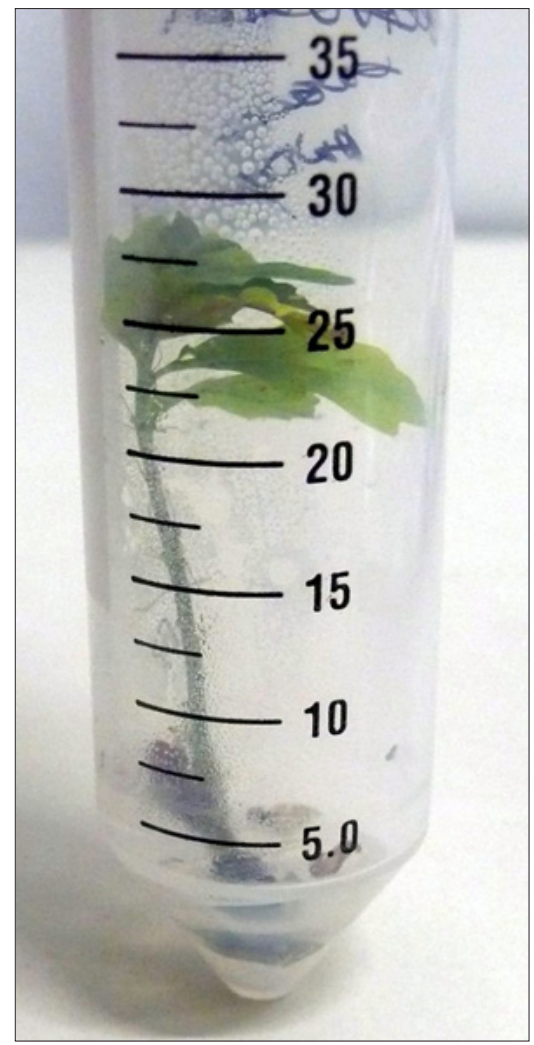

Figure 5: First signs of chlorosis after 45 days of culture. 